\title{
Research
}

\section{Use of medication and investigations in community hospitals and a district general hospital: a cohort study of emergency admissions in the elderly}

Sally Stapley Research Manager, Caper Research Practices, Exeter, UK, Tania Crabb Research Assistant, Caper Research Practices, Exeter, UK, Alison Round Director of Public Health, East Devon Primary Care Trust, Exeter, UK, Sandra Hollinghurst Health Economist, University of Bristol, Bristol, UK, William Hamilton Senior Research Fellow, University of Bristol, Bristol, UK

Background: We previously studied clinical outcomes in two cohorts of emergency admissions of patients aged over 70 to one district general hospital (DGH) or five community hospitals (CHs) finding no differences in death, readmissions, or quality of life at 6 months after admission. Our objectives in this study were to examine the use and cost of medication and use and cost of investigations in the two different hospital settings.

Methods: We identified and priced all medication taken on admission, during the hospital stay, and on discharge, and identified all investigations. The main outcome measures were number and cost of medications and investigations during the stay, and the difference in the patients' routine medication before and after the hospital stay.

Results: Three hundred and seventy five patients were studied (DGH 170, $\mathrm{CH} 205$ ). The median (inter-quartile range (IQR)) number and cost of drugs used during the hospital stay was higher in DGH patients: $11(8,17)$ drugs than $\mathrm{CH}$ patients: $8(5,11) ;(P<0.001)$, costing $£ 35(13,90)$ and $£ 16(6,45)$ respectively $(P<0.001)$. DGH patients had a mean of 0.55 drugs added to their routine medication, at an extra projected cost of $£ 9.50$ per month, whereas $\mathrm{CH}$ patients had an average of 0.11 drugs removed from their routine medication at a projected cost saving of $f 1.35$ per month $(P=0.012$ for number and $<0.001$ for cost). More investigations were performed in the $\mathrm{DGH}$ than the $\mathrm{CH}$; DGH median (IQR) $£ 260(180,410)$; $\mathrm{CH}$ median $£ 40(9,110)$. Conclusions: Considerable extra resources are used in emergency admissions of the elderly to a DGH. However, these extra resources do not appear to yield mortality or morbidity benefits.

Key words: community hospital; emergency admission; prescribing

Received: March 2006; accepted: October 2006

\section{Background}

Emergency hospital admissions are rising by $2-3 \%$ each year and account for $40 \%$ of acute National

\footnotetext{
Address for correspondence: Sally Stapley, Research Manager, Caper Research Practices, The Dairy, Stoke Hill Farm, Exeter EX4 5BW, UK. Email: Sal.Stapley@Exeter-PCT.nhs.uk
}

(C) 2007 Cambridge University Press
Health Service (NHS) bed use (Capewell, 1996). More than two thirds of emergency admissions are of elderly patients (Edwards and Hensher, 1998). Overall, in-patient bed numbers have not increased, so pressure has mounted on hospitals to accelerate turnover and reduce admission rates (National Audit Office, 2003). Alternative methods of healthcare such as community hospitals (CHs), nursing 
homes, and 'hospital at home' schemes may be able to meet some of the demand for emergency care (Department of Health, 2001). Recent governmental initiatives propose increasing the $\mathrm{CH}$ sector.

Currently, most emergency admissions are to district general hospitals (DGHs). In more rural parts of the UK, there are also CHs (Young and Donaldson, 2001). Medical care in CHs is usually provided by general practitioners (GP). There is less access to complex investigations and variable access to other health professionals, such as visiting consultants. In contrast, DGHs have medical teams of a consultant and junior doctors, and a wide range of investigations available.

There is little research on costs of CHs (Young and Donaldson, 2001), most reports describing clinical features of patients admitted to a $\mathrm{CH}$ (Boston et al., 2001; Seamark et al., 2001). Economic analyses have been hampered by the different case-mix in the two settings (Young and Donaldson, 2001), and have largely examined single conditions, such as dental or cataract surgery (Haynes et al., 2001; Panula et al., 2002). One modelling study estimated the costs of stroke care between CHs and DGHs, suggesting total costs may be up to $60 \%$ lower in the $\mathrm{CH}$ (Henderson and Scott, 2001). A Dutch study calculated the daily cost in one $\mathrm{CH}$ to be $67 \%$ of the cost in a DGH (Hakkaart-van Roijen et al., 2004). In the Dutch study the lengths of stay and clinical outcomes were not studied, but assumed to be similar.

This study aimed to compare the use of medication and investigations in $\mathrm{CHs}$ and a DGH and evaluate the cost differences from the perspective of the NHS. We studied length of stay, number and cost of investigations, and use of medication in two cohorts of emergency admissions to a DGH and five $\mathrm{CHs}$ known to have similar clinical outcomes.

\section{Methods}

This is a secondary analysis of a study examining the clinical outcomes of emergency admissions for patients aged over 70 years to either one DGH or one of five CHs in Devon, UK, between June 1999 and November 2000 (Round et al., 2004). Patients were eligible if their condition could have been managed equally well in either hospital. Patients were excluded if they required specific hospital admission, such as gastro-intestinal bleeding for the DGH or palliative care for the CHs. It was not a randomized-controlled trial as it was considered unethical to admit an elderly ill patient to a hospital up to 20 miles away from their home when one was available nearby.

A total of 376 patients were recruited, but one had incomplete medication data, so this analysis is of 375 patients (DGH 170, $\mathrm{CH} 205$ ).

\section{Demographic and clinical features of the two cohorts}

On admission, the following measures of functional state and mental health were taken using standard or validated questionnaires: Activities of Daily Living (ADL) (Katz et al., 1963), MiniMental State Examination (MMSE) (Folstein et al., 1975), EuroQol 5D (Kind et al., 1994), SF-36 (Walters et al.,2001) and co-morbidity index (Incalzi et al., 1997).

Overall both cohorts had poor health, with substantial co-morbidity and moderately impaired functional ability. The outcomes of the cohorts 6 months after admission were: mortality, place of residence, readmissions and quality of life. Between admission and follow-up at 6 months 122 patients (DGH 52, CH 70) were lost to follow-up through death or withdrawal from the study. The number of patients with recorded outcome measures 6 months after admission is 254 (DGH 118, CH 136).

The characteristics of the cohorts on admission and their clinical outcomes are summarized in Table 1. This information is included to highlight the similarities between the two cohorts. The only significant clinical or demographic difference between the cohorts on admission was that the $\mathrm{CH}$ patients were slightly older, at a median age of 84 years, compared with 81 years in the DGH cohort. Six months after discharge there were no significant differences between the cohorts.

The initial hospital diagnoses were recorded from the written notes and grouped into broad categories. There was a difference in the diagnostic labels given to the two cohorts, with respiratory and cardiovascular diagnostic labels more common in the DGH cohort and miscellaneous labels more common in the $\mathrm{CH}$ cohort. This difference was considered to be partly artefactual as GPs in the $\mathrm{CH}$ were admitting to their own beds, whereas in the DGH admissions a 'formal' label had to be given to ensure the admission was accepted. It is unlikely 
Table 1 Characteristics on admission and outcome in the two cohorts

\begin{tabular}{llll}
\hline Admission comparison & $\begin{array}{l}\text { DGH } \\
n=170\end{array}$ & $\begin{array}{l}\mathrm{CH} \\
n=205\end{array}$ & $P$-value \\
\hline Number (\%) male & $77(45 \%)$ & $71(35 \%)$ & 0.036 \\
Median age (IQR) & $81(76-85)$ & $84(79-88)$ & $<0.001$ \\
Median MMSE (IOR) & $17(16-18)$ & $17(16.9-18)$ & 0.69 \\
Median co-morbidity (IOR) & $5(4-5)$ & $4(4-5)$ & 0.52 \\
Median ADL score (IOR) & $10(10-10)$ & $9(9-10)$ & 0.06 \\
Median EuroQol score (IOR) & $0.26(0.19-0.36)$ & $0.26(0.15-0.31)$ & 0.82 \\
Median length of hospital stay (IOR) & $12(8-22)$ & $14(7-24)$ & 0.66 \\
Outcome at 6 months & $n=118$ & $n=136$ & \\
Mortality (\%) & $22(18.8 \%)$ & $23(17 \%)$ & 0.48 \\
Nursing or residential home admission (\%) & $3(2.5 \%)$ & $10(7.4 \%)$ & 0.76 \\
Readmissions (\%) & $17(14.4 \%)$ & $15(11 \%)$ & 0.41 \\
Mean change in EuroQol score (CI) & $6.5(2.4-10.7)$ & $6.6(2.8-10.4)$ & 0.97 \\
Mean change in SF-36 score (Cl) & $0.22(-2.6-3.0)$ & $0.13(-2.3-2.5)$ & 0.97 \\
\hline
\end{tabular}

that the differences in diagnostic labelling are a major source of bias, given the similar functional status between the two groups.

\section{Data collection}

\section{Medication}

Prescribed medication on admission was identified from the nursing and medical records. Medication prescribed during the admission was taken from the drug chart, and discharge medication from the discharge prescription. A cost was attributed to each medication using the British National Formulary September 1999, current during the course of the study. The British National Formulary was preferred to the NHS Drug Tariff as it was more comprehensive. For all items issued as a unit, such as inhalers or tubes of skin products, we assumed a single item was used per month or during the hospital stay. For other medications, we calculated the actual cost of the drugs issued. During the patients' stay in hospital this included antibiotics and analgesics, but not intravenous fluids. A projected 28-day cost for admission and discharge drugs was calculated, on the assumption that these would be continued long-term. Antibiotics and analgesics were excluded from the admission and discharge calculations, as they were expected to be used in a short course or intermittently. They were retained for analysis of costs during the hospital stay.

\section{Investigations during hospital stay}

All investigations performed during the admission were identified. For blood tests, multi-channel investigations (eg, full blood count) counted as one investigation. Investigations were analysed by volume and cost within four categories: chemical pathology, haematology, radiology and other. All investigations, apart from radiology, were analysed at the DGH laboratory. At the time of the study, $\mathrm{CHs}$ were charged a fixed annual fee from the DGH for these tests, irrespective of the actual number ordered. For this study, we assumed the cost to the NHS of an individual test was the same for all investigations irrespective of its setting. For radiology, each hospital provided their own costs for inhouse tests: for tests such as computed tomographic (CT) scanning in $\mathrm{CH}$ patients (which will have been performed in the DGH), the DGH cost was used.

\section{Statistical analysis}

Sex and diagnosis on admission were compared using a Pearson $\chi^{2}$ test. Age, duration of hospital stay, number and cost of drugs were not normally distributed, so were compared using Wilcoxon ranksum tests. Regression models were constructed with age, sex and hospital setting as possible explanatory variables. The difference in number and cost of drugs between admission and discharge was normally distributed, so comparison was by means and $t$-tests. All analyses were performed in STATA, version 8 (StataCorp, 2001).

Primary Health Care Research \& Development 2007; 8: 105-111 


\section{Results}

The cohorts are described in the Methods section and Table 1.

\section{Medication}

Use of medication during the hospital stay is shown in Table 2. The median total number and cost of drugs prescribed was significantly higher in the DGH. However, use of antibiotics and analgesics was not higher in the DGH. In multi-variable regressions including sex, gender and hospital setting, hospital setting was the only variable significantly associated with the total cost of medication $(P=0.009)$.

Drug use on admission to and discharge from hospital is summarized in Table 3. Patients admitted to the DGH were taking a larger number of separate drugs than those admitted to the $\mathrm{CH}$, though the difference in projected 28-day cost was

Table 2 Use of medication during the hospital admission in the two cohorts

\begin{tabular}{llll}
\hline $\begin{array}{l}\text { During } \\
\text { hospital stay }\end{array}$ & $\begin{array}{l}\text { DGH median } \\
(\mathrm{IQR})\end{array}$ & $\begin{array}{l}\mathrm{CH} \text { median } \\
(\mathrm{IQ})\end{array}$ & $P$-value \\
\hline $\begin{array}{l}\text { Total number of } \\
\text { drugs per patient }\end{array}$ & $11(8-17)$ & $8(5-11)$ & $<0.001$ \\
$\begin{array}{l}\text { Antibiotics } \\
\text { Analgesics }\end{array}$ & $1(0-2)$ & $1(0-2)$ & 0.19 \\
Other & $1(0-2)$ & $1(0-2)$ & 0.59 \\
Total cost of & $9(6-13)$ & $6(4-8)$ & $<0.001$ \\
drugs per & $(12.6$ & 15.8 & $<0.001$ \\
patient $(\mathrm{f})$ & & $(5.80-45.60)$ & \\
Antibiotics & $0.21(0-16.60)$ & $0.14(0-3.20)$ & 0.09 \\
Analgesics & $0.1(0-0.60)$ & $0.1(0-0.80)$ & 0.58 \\
Other & 26.9 & 11.6 & $<0.001$ \\
& $(11.40-54.40)$ & $(4.00-35.30)$ & \\
\hline
\end{tabular}

of borderline significance. On discharge, the DGH patients were again taking a higher number of separate drugs, but at a considerably higher projected 28-day cost. In multi-variable regressions including sex, gender and hospital setting, hospital setting was the only variable significantly associated with the difference in total cost of medication $(P<0.001)$.

In the DGH cohort, 34 (20\%) of patients were discharged with fewer drugs than they were taking on admission, compared with $68(33 \%)$ of patients in the $\mathrm{CH}$ cohort. In contrast, $98(58 \%)$ of patients in the DGH cohort were discharged with increased medication compared with 69 (34\%) from the $\mathrm{CH}$ cohort.

Although the distribution of the above variables required the use of medians and non-parametric tests for statistical analysis, mean costs can be useful when calculating total costs. These were as follows: drugs per patient during hospital stay, DGH $£ 102.74$ (standard deviation (SD) £294.69), CH $£ 41.86$ (£82.61): prescribed items per patient on admission, DGH £24.38 (£23.30), CH £20.56 (£23.24): prescribed items per patient on discharge, DGH $£ 33.89$ (£30.57), CH 19.20 (£21.34).

\section{Investigations}

The number and cost of all investigations was significantly higher in the DGH than the $\mathrm{CH}$ (Table 4). In multi-variable regressions including sex, gender and hospital setting, hospital setting was the only variable significantly associated with either the number or the cost of investigations $(P<0.001$ in both regressions).

Although the distribution of the above variables mandated the use of median and non-parametric tests, the mean values for the total cost (SD) of investigations during hospital stay per patient is DGH £323.94 (£245.73), CH £65.94 (£74.27).

Table 3 Number and projected 28-day costs for drugs on admission and discharge

\begin{tabular}{|c|c|c|c|}
\hline & DGH & $\mathrm{CH}$ & $P$-value \\
\hline Median (IQR) number of prescribed items on admission per patient & $4(3-6)$ & $3(2-6)$ & 0.038 \\
\hline Median (IQR) cost of prescribed items on admission per patient $(\mathrm{f})$ & $22.7(7.80-40.10)$ & $17.8(3.70-37.40)$ & 0.085 \\
\hline Median (IQR) number of items prescribed on discharge per patient & $5(3-7)$ & $4(2-6)$ & $<0.001$ \\
\hline Median (IQR) cost of items prescribed on discharge per patient $(\mathrm{f})$ & $30.5(11.40-53.30)$ & $16.9(4.30-30.90)$ & $<0.001$ \\
\hline Mean (SD) change in number of drugs per patient & $0.55(2.8)$ & $-0.12(2.33)$ & 0.012 \\
\hline Mean (SD) change in cost of drugs per patient $(\mathrm{f})$ & $9.5(29.60)$ & $21.4(22.80)$ & $<0.001$ \\
\hline
\end{tabular}

Note: Analgesics and antibiotics excluded from all analyses.

Primary Health Care Research \& Development 2007; 8: 105-111 


\section{Discussion}

\section{Main findings}

In two cohorts of emergency admissions of the elderly, recruited simultaneously to either a $\mathrm{CH}$ or a DGH, there was a higher level of intervention in the DGH, both for investigations and for prescribed medication. Fewer drugs were used in the $\mathrm{CH}$, and the drugs chosen were less expensive. Furthermore, alterations in the patients' regular medication were much greater in patients who had been admitted to the DGH.

\section{Limitations of the study}

This was not a randomized-controlled trial, which would have been the ideal method of study. However, such a study design would have been unethical. Nonetheless, the two cohorts were broadly similar (reflecting the strict eligibility criteria). The $\mathrm{CH}$ patients were older, but were taking fewer drugs on admission. Despite these small differences on admission, both cohorts had the same outcomes, as measured by death, readmission, and place of residence after admission and quality of life. Our cost calculations ignored the actual procurement policies in place: this was deliberate, as if expansion of intermediate care takes place, it would lead to real changes in costs, and would probably provoke a change in inter-hospital financial arrangements.

Why should such a striking difference in use of drugs and investigations have occurred? We consider it unlikely that it reflects differences in the diagnostic categories (as we suspect these are artefactual), though it is possible that the higher proportion of patients in the DGH labelled with respiratory or cardiac conditions could have impacted on costs. The first likely reason is availability. In the $\mathrm{CHs}$, there is a smaller range of drugs routinely available, with the range restricted to commonly used drugs. These are generally cheaper. This may partly explain the higher cost of drugs during admission, but is unlikely to explain the higher number of drugs prescribed in the DGH, eleven compared to eight in the CHs. We did not measure any discounting that the hospitals may have obtained for bulk purchases of drugs, so it is possible that the cost difference between the $\mathrm{DGH}$ and the $\mathrm{CHs}$ would be reduced by a greater purchasing power in the DGH. This would not apply to discharge drugs, as they will be prescribed by the GP, and dispensed by a local pharmacy. Furthermore, some complex investigations such as CT scanning require transporting the patient to the DGH. This issue may raise the threshold for ordering such an investigation.

A second possible explanation is the different make-up of the medical teams. In the $\mathrm{CHs}$, medical care is given by the patient's own GP, or by a doctor from the same general practice. This doctor's priority may simply be to treat the patient's recent illness, with little thought given to searching for, let alone treating, co-morbidity. This could explain the small number of investigations, and small number of changes to regular medication. In the $\mathrm{DGH}$, however, there is constant pressure on medical wards to free up bed space. There is an onus on staff, such as junior hospital doctors, to make use of resources to effect an early discharge. Despite this, length of stay in hospital was only related to age, with the hospital setting insignificant. It is also likely that the medical care in the DGH is more protocol-based.

Table 4 Use of investigations during the hospital admission in the two cohorts

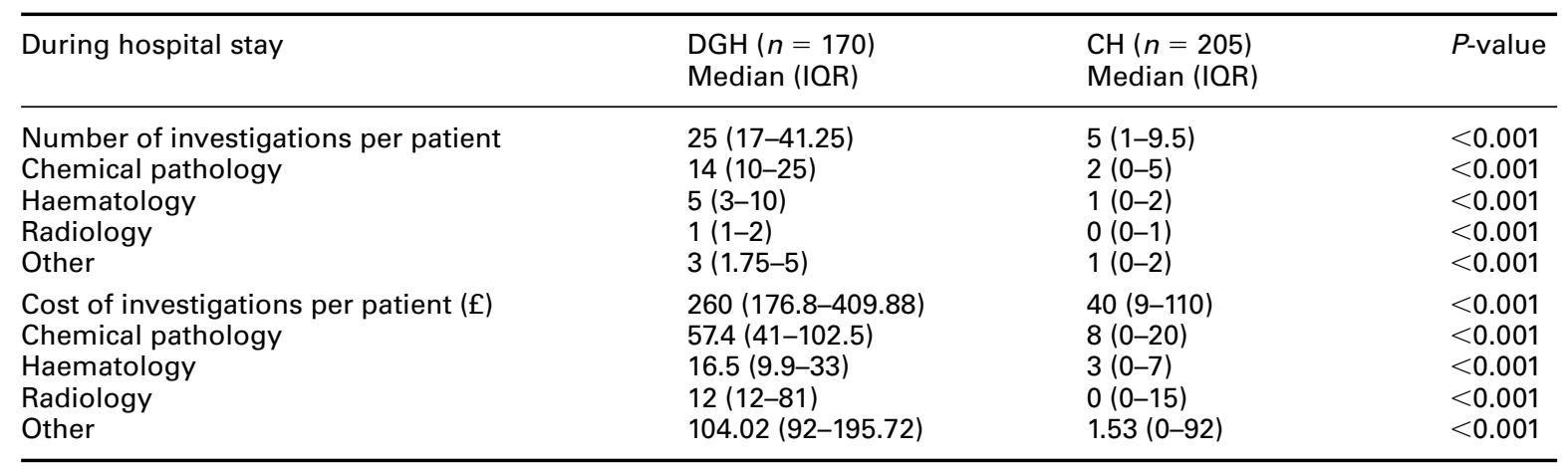


This could have increased the use of investigations in particular. Furthermore, junior hospital doctors may practise a more defensive style of medicine than the experienced GP, and thus order more tests to ensure treatable illness is not missed.

\section{Conclusions}

The overall cost differences are relatively small when compared with the capital and staffing costs of the two hospital settings. Nonetheless, given that both cohorts had similar outcomes, some of the additional investigations and changes to regular medication are questionable. On average, changes in regular medication increased the recurring monthly cost of the patient's medication by over $£ 10$, let alone the possibility of confusing elderly patients (and their doctor's practices) with all the changes. However, a positive message can be read into our results: that care of selected elderly admissions in $\mathrm{CHs}$ provides a good alternative to DGH care. With the clinical outcomes being similar (albeit in this selected group of patients) and the lengths of stay being similar, policy can be driven by considerations of cost, and by the convenience to patients and their relatives of admission closer to their homes. Our results therefore support recent UK policy initiatives to increase $\mathrm{CH}$ use (Department of Health, 2006). Medical care in them will generally be provided by GPs (which has benefits of continuity of care over and above the fiscal benefits seen in this study). However, medical staffing does not necessarily have to be provided by primary care. This flexibility can again be seen positively: if GPs wish to offer hospital care, they may have more opportunities for doing so. Equally, if they prefer not to do so, they are increasingly able to opt out.

\section{Competing interests}

The authors declare that they have no competing interests.

\section{Authors' contributions}

SS and TC collected the data; SS and WH analysed it. AR, WH, and SH designed the study. All authors Primary Health Care Research \& Development 2007; 8: 105-111 contributed to preparation of the manuscript, and have read and approved it.

\section{Acknowledgements and funding}

The study was funded by a grant from the Charitable Trustees of North and East Devon Health Authority. North and East Devon Local Research Ethics Committee approved the study and all patients recruited gave informed consent.

\section{References}

Boston, N.K., Boynton, P.M. and Hood, S. 2001: An inner city GP Unit versus conventional care for elderly patients: prospective comparison of health functioning, use of services and patient satisfaction. Family Practice 18, 141-48.

Capewell, S. 1996: The continuing rise in emergency admissions. British Medical Journal 312, 991.

Department of Health. 2001: Shaping the future NHS: Long term planning for hospitals and related services response to the consultation exercise on the findings of the National Beds Inquiry. Department of Health: London.

Department of Health. 2006: Our health our care our say, HM government. Department of Health: London.

Edwards, N. and Hensher, M. 1998: Managing demand for secondary care services: the changing context. British Medical Journal 317, 135-38.

Folstein, M.F., Folstein, S.E. and McHugh, P.R. 1975: Minimental state: a practical method for grading the cognitive sate of patients for the clinician. Journal of Psychiatric Research 12, 189-98.

Hakkaart-van Roijen, L., Moll van Charante, E., Bindels, P., Yzermans, C. and Rutten, F. 2004: A cost study of a general practitioner hospital in the Netherlands. European Journal of General Practice 10, 46-49.

Haynes, R., Gale, S., Mugford, M. and Davies, P. 2001: Cataract surgery in a community hospital outreach clinic: patients' costs and satisfaction. Social Science and Medicine 53, 1631-40.

Henderson, L.R. and Scott, A. 2001: The costs of caring for stroke patients in a GP-led community hospital: an application of programme budgeting and marginal analysis. Health and Social Care in the Community 9, 244-54.

Incalzi, R.A., Capparella, O., Gemma, A., Landi, F., Bruno, E., Di Meo, F. and Carbonin, P. 1997: The interaction between age and comorbidity contributes to predicting the mortality of geriatric patients in the acute care hospital. Journal of Internal Medicine 242, 291-98.

Katz, S., Ford, A.B., Moskowitz, R.W., Jackson, B.A. and Jaffe, M.W. 1963: Studies of illness in the aged. The index of ADL: a standardized measure of biological and psychosocial function. Journal of the American Medical Association 185, 914-19. 
Kind, P., Gudex, C., Dolan, P. and Williams, A. 1994: Practical and methodological issues in the development of the EuroQol. The York experience. Advances in Medical Sociology, Volume 5. Quality of Life in Health Care. J.A.I. Press.

National Audit Office. 2003: Ensuring the effective discharge of older patients from NHS acute hospitals. Stationery Office: London.

Panula, K., Keski-Nisula, L., Keski-Nisula, K., Oikarinen, K. and Keski-Nisula, S. 2002: Costs of surgical-orthodontic treatment in community hospital care: an analysis of the different phases of treatment. International Journal of Adult Orthodontics and Orthognathic Surgery 17, 297-306.

Round, A., Crabb, T., Buckingham, K., Mejzner, R., Pearce, V., Ayres, R., Weeks, C. and Hamilton, W. 2004: Six month outcomes after emergency admission of elderly patients to a community or a district general hospital. Family Practice 21, 173-79.

Seamark, D., Moore, B., Tucker, H., Church, J. and Seamark, C. 2001: Community hospitals for the new millennium. British Journal of General Practice 51, 125-27.

StataCorp. 2001: Stata Statistical Software: Release 8.0. Stata Corporation: College Station, TX.

Walters, S.J., Munro, J.F. and Brazier, J.E. 2001: Using the SF36 with older adults: a cross-sectional community based survey. Age and Ageing 30,337-43.

Young, J. and Donaldson, K. 2001: Community hospitals and older people. Age and Ageing 30-S3, 7-10. 